Revue des patrimoines

$20 \mid 2013$

Les patrimoines de la traite négrière et de l'esclavage

\title{
Loyola, l'habitation des jésuites de Rémire en Guyane française
}

\section{Yannick Le Roux}

\section{(2) OpenEdition}

\section{Journals}

Édition électronique

URL : http://journals.openedition.org/insitu/10170

DOI : 10.4000/insitu.10170

ISSN : 1630-7305

Éditeur

Ministère de la Culture

Référence électronique

Yannick Le Roux, «Loyola, I'habitation des jésuites de Rémire en Guyane française », In Situ [En ligne], 20 | 2013, mis en ligne le 14 février 2013, consulté le 14 novembre 2019. URL : http:// journals.openedition.org/insitu/10170; DOI : 10.4000/insitu.10170

Ce document a été généré automatiquement le 14 novembre 2019.

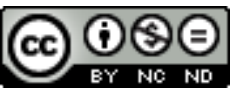

In Situ Revues des patrimoines est mis à disposition selon les termes de la licence Creative Commons Attribution - Pas d'Utilisation Commerciale - Pas de Modification 4.0 International. 


\title{
Loyola, l'habitation des jésuites de Rémire en Guyane française
}

\author{
Yannick Le Roux
}

Figure 1

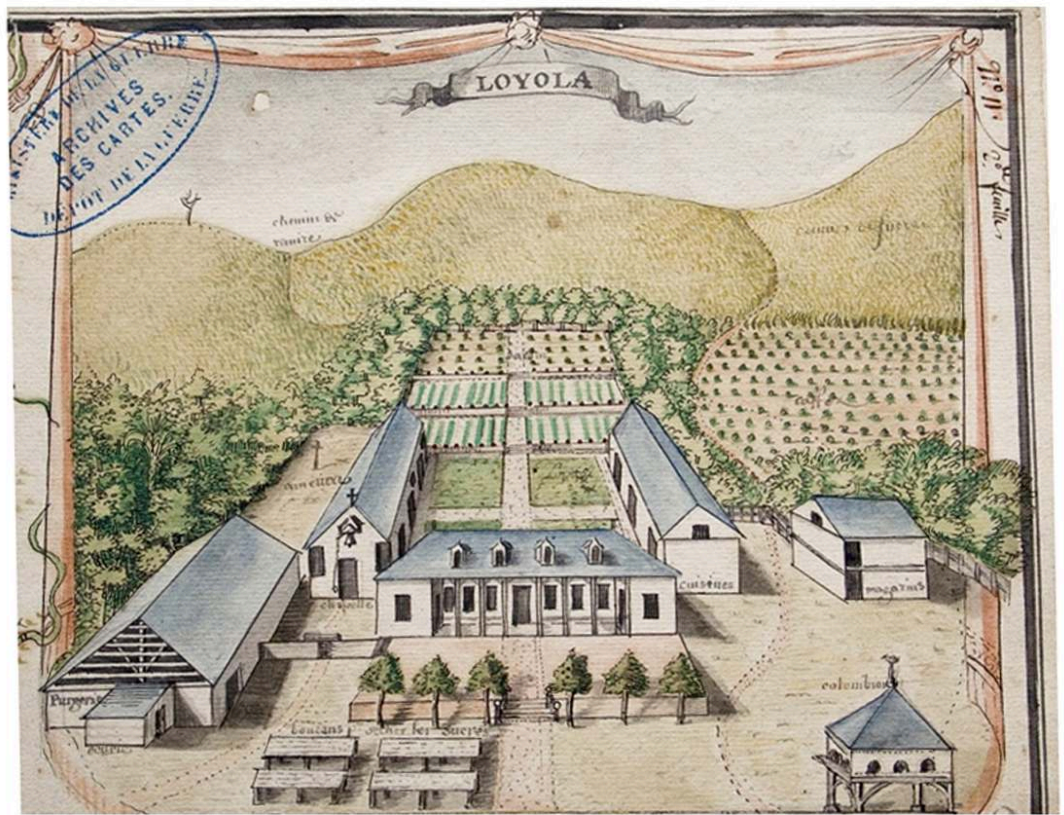

Vue cavalière de l'habitation Loyola par Hébert 1730 (SHAT Vincennes) (c) A. Le Roux.

L'habitation des jésuites de Guyane, l'habitation Loyola, a été fondée en 1668 (fig. nº1). Les religieux, nouvellement arrivés en Amérique pour la conversion des Sauvages, 
avaient besoin d'avoir des revenus réguliers et importants pour financer leurs missions auprès des Amérindiens. Les habitations du Mont-Xavier à Kourou, de Maripa et de Saint-Régis, sur la Comté, enfin celles de Mont-Louis et de Loyola à Rémire, répondaient à cette nécessité économique. Très rapidement, leur grande maîtrise de l'économie coloniale allait leur donner la première place dans ce domaine. Leur position matérielle était confortée localement par leur monopole sur toutes les affaires religieuses de la Guyane. Ainsi, ils assumaient seuls la charge spirituelle de toute la colonie : missions amérindiennes, desserte des paroisses, catéchèse des colons et des esclaves, enseignement. Pour l'évangélisation et la protection des Amérindiens, ils avaient obtenu de limiter l'accès, et encore plus l'installation des colons, dans les immenses territoires des missions, laissant aux habitants une région réduite à une étroite bande de terrains côtiers, de l'Oyapock au fleuve Kourou. Il n'est pas exagéré de considérer que, pendant toute la première moitié du XVIII ${ }^{e}$ siècle, les jésuites étaient devenus les véritables maîtres de la Guyane (fig. $\mathbf{n}^{\circ} \mathbf{2}$ ).

Figure 2

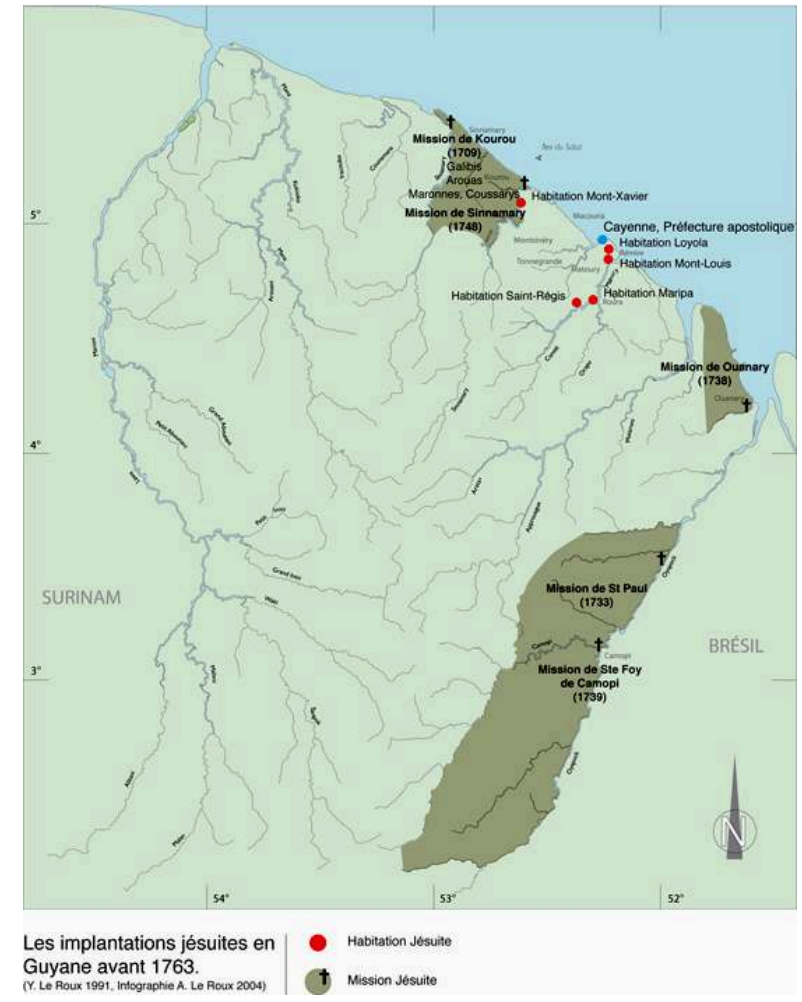

Carte de la présence jésuite en Guyane au XVIIIe siècle.

Infographie Aurélien Le Roux. (c) A. Le Roux.

2 L'habitation Loyola a été constituée, au départ, par la réunion de deux concessions qui remontent à la première période de l'histoire coloniale de la Guyane : celle du juif hollandais (1654-1666) Isaac Drago et le terrain de l'habitation de Pinon de Quincy. Commencé en 1668, le domaine des jésuites sera, par la suite, considérablement agrandi par des acquisitions, des donations, des concessions, pour atteindre, à son apogée, une superficie de plus de 1000 ha (fig. $\mathbf{n}^{\circ} \mathbf{3}$ ). 
Figure 3

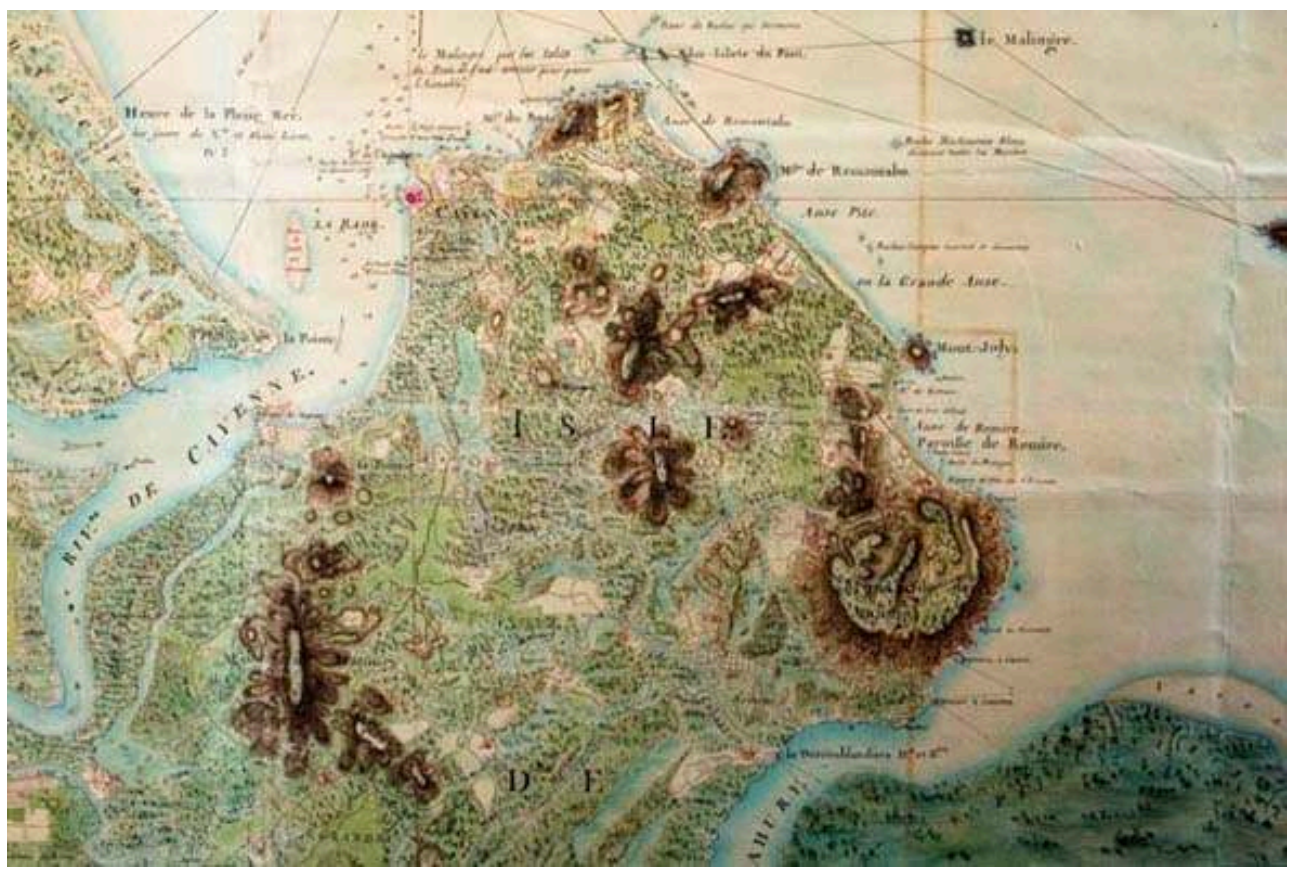

Carte de Desingy, 1771, montrant, en couleurs, l'emprise du domaine de Loyola.

(c) A. Le Roux.

3 Même si les documents retrouvés, portant sur l'activité de cet établissement, déplorent fréquemment la médiocrité de ses revenus, l'importance des productions était tout de même à la mesure de cette immense habitation. Dans un pays marqué, à cette époque, par le marasme économique, la sucrerie des jésuites sera ainsi et de très loin, la première de la Guyane pendant un quart de siècle (C. 1720-1753). La récolte du café et du cacao représentait, quant à elle, la moitié de la quantité produite dans toute la colonie et la grande poterie, ainsi que la forge de Loyola, fournissaient également en produits Cayenne et les petites habitations de la région. Ces activités artisanales procuraient aux jésuites d'importants revenus complémentaires.

4 Reflet de la position éminente des religieux en Guyane, la maison de maître de l'habitation sera le lieu de séjour de personnages importants comme le savant académicien Charles-Marie de La Condamine. En 1744, au retour de son expédition dans les Andes pour mesurer le méridien terrestre, il y résida pour se livrer à quelques expériences et réaliser la carte topographique de l'île de Cayenne. En 1762, Christophe Fusée-Aublet séjourna, à son tour, à Loyola où il effectua ses premiers travaux destinés à son grand ouvrage de botanique: Histoire des plantes de la Guyane françoise, édité en 1775.

5 En 1762, un arrêt du Roi interdisait la Compagnie de Jésus, en France et dans ses colonies. Au surplus, déclaré en faillite, l'ordre fut condamné au remboursement de ses dettes; ses possessions, dont Loyola, furent alors saisies pour rembourser les créanciers. Au moment d'être chassés de la colonie, on procéda à l'évaluation de leurs biens. On constate, à cette occasion, que la dimension de l'établissement était impressionnante, d'ailleurs personne ne fut en mesure de payer les 200000 Livres de son estime. Si l'on se fie à l'usage colonial qui mesurait la «force » d'une habitation au nombre d'esclaves qui y travaillaient, on n'y dénombrait pas moins de 500 esclaves, 
chiffre tristement indépassable, même dans les plus puissantes plantations de SaintDomingue.

6 Singularité de cette habitation esclavagiste, son rôle spirituel, matérialisé par la présence d'une chapelle et d'un cimetière, était loin d'être négligeable. On sait que les jésuites rencontrèrent un réel succès dans l'évangélisation des esclaves, beaucoup plus, en tout cas, qu'auprès des populations amérindiennes qui étaient pourtant au cœur de leur projet missionnaire. À maintes reprises, cette modeste chapelle jouera également le rôle d'église paroissiale où nombre de colons ou d'Amérindiens convertis étaient baptisés, s'y mariaient et y avaient parfois leur sépulture. Quelques grands habitants avaient le privilège d'être inhumés dans la chapelle.

7 Après le départ des jésuites, cette habitation sera abandonnée. Le terminus post quem, maintes fois confirmé par les observations archéologiques, se situe vers 1768, date du départ du dernier jésuite et du transfert des activités vers Beauregard, nouvel établissement construit à deux kilomètres au sud. Désaffecté, ce site sera laissé, sans aucun remaniement postérieur, à une envahissante forêt qui jouera un rôle protecteur pour ses vestiges. Même si, au XIX ${ }^{e}$ siècle, des prélèvements de matériaux ont quelque peu affecté ces ruines, ces ponctions n'ont en rien altéré la lisibilité de ce témoignage, si important de l'histoire de la Guyane, qui se présente aujourd'hui avec une grande intégrité archéologique (fig. $\left.\mathbf{n}^{\circ} \mathbf{4}\right)$.

Figure 4

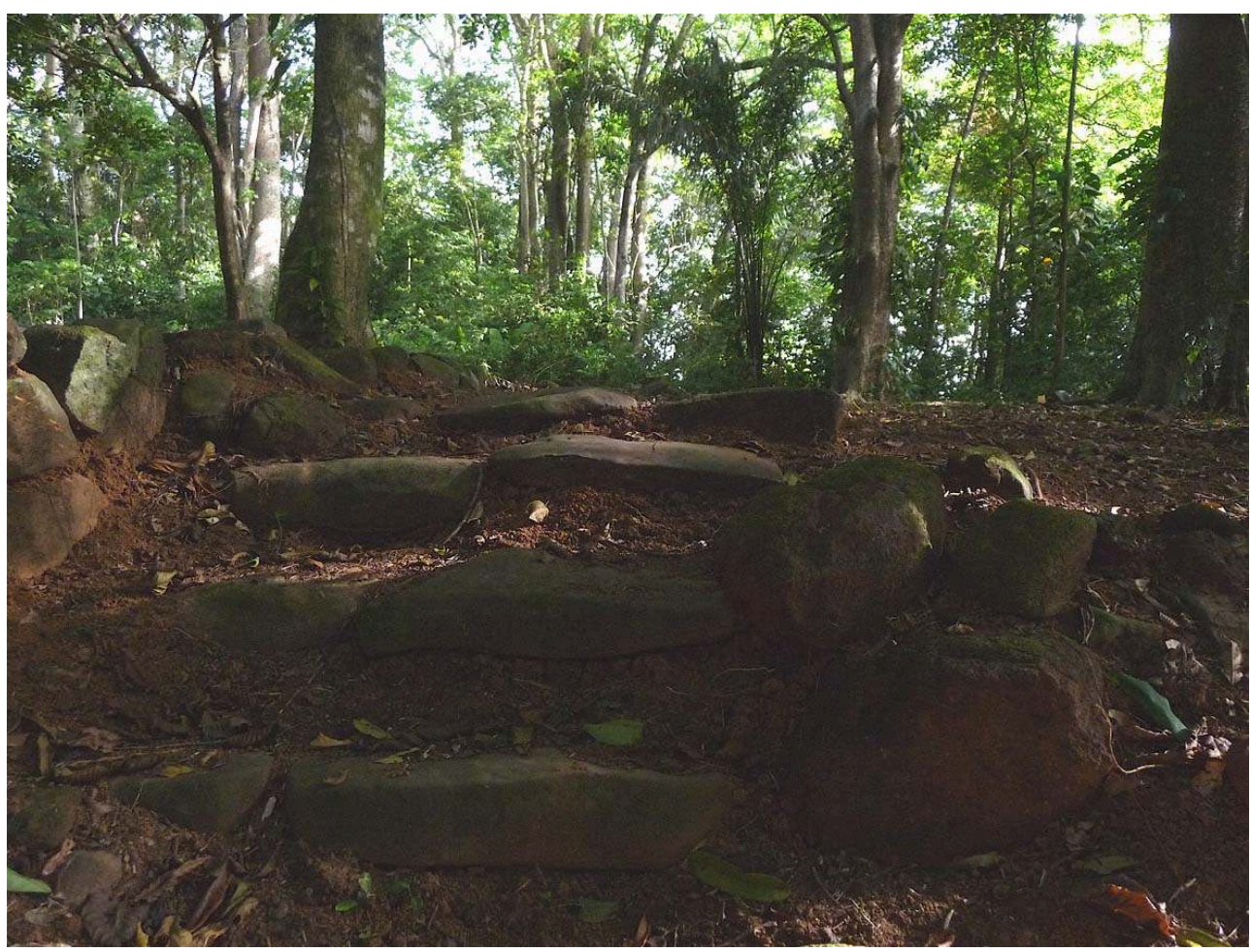

Les vestiges de l'habitation ont été retrouvés enfouis dans une épaisse forêt de régénération.

Phot. Y. Le Roux. (c) Y. Le Roux.

Les vestiges découverts de cette grande habitation peuvent être regroupés en plusieurs secteurs : résidentiel, religieux, artisanal et industriel. À l'heure actuelle, une grande partie du site reste encore à découvrir et à étudier. 


\section{Le secteur résidentiel}

Il est dominé par la maison de maître, vaste bâtiment de 24 mètres de long sur 10 mètres de large. Cette construction a été établie sur la première terrasse d'un ensemble qui en compte cinq. Ces terrassements, soutenus par de gros murs, s'étagent sur le flanc de la montagne des Jésuites. Ils sont accessibles par des escaliers et une allée qui les traverse en leur centre, établissant une distribution symétrique des constructions dans la tradition du jardin à la française. Les fouilles ont permis de retrouver le sol originel de cette maison, avec les pavements, les cloisons et l'emplacement de l'escalier. Les murs en charpente, hourdis en torchis, ont disparu et la toiture, couverte en bardeaux de bois dur, n'a pas laissé de traces. Cette construction, datable des années 1720 , représente certainement le plus ancien exemplaire de maison créole en charpente retrouvé à ce jour (fig. $\mathbf{n}^{\circ}$ ).

Figure 5

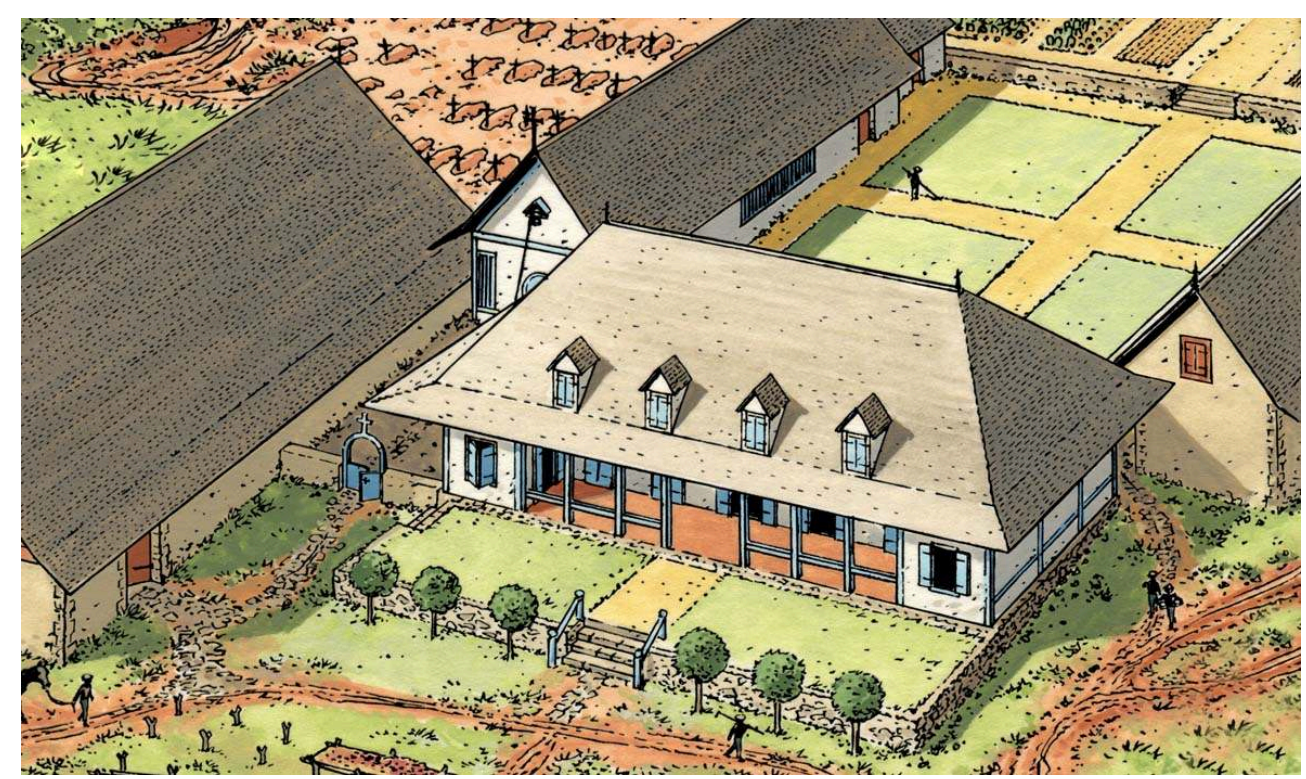

Restitution de la maison de maître des jésuites par Patrice Pellerin (2002).

(c) P. Pellerin.

10 En arrière de la maison de maître s'étend une vaste cour carrée de 24 mètres de côté. Elle est traversée par deux allées qui se croisent en son centre. Cette cour devait servir au rassemblement des esclaves pour les cérémonies religieuses. Outre la maison de maître, elle est encadrée par deux corps de bâtiment : la chapelle, à l'est et la cuisine hôpital, à l'ouest.

11 La cuisine est construite sur un plan carré de 6 mètres de côté. La fouille a permis de mettre en valeur un four à pain, l'âtre d'une grande cheminée et une petite construction à usage de cuisinière (le potager).

12 Adossé à la cheminée, un mur de refend sépare la cuisine d'une pièce attenante, de 12 mètres de long, à usage d'hôpital. Cette disposition permettait de diffuser la chaleur de la cuisine dans l'hôpital; elle est à relier aux croyances médicales anciennes qui attribuaient à la chaleur des vertus thérapeutiques. La présence d'un tel hôpital, voué 
aux soins des esclaves, aux abords immédiats de la maison de maître, n'est pas fréquente dans le contexte esclavagiste. On peut mettre ce choix au crédit de la vocation « charitable » des religieux (fig. $\mathbf{n}^{\circ} \mathbf{6}$ ).

Figure 6

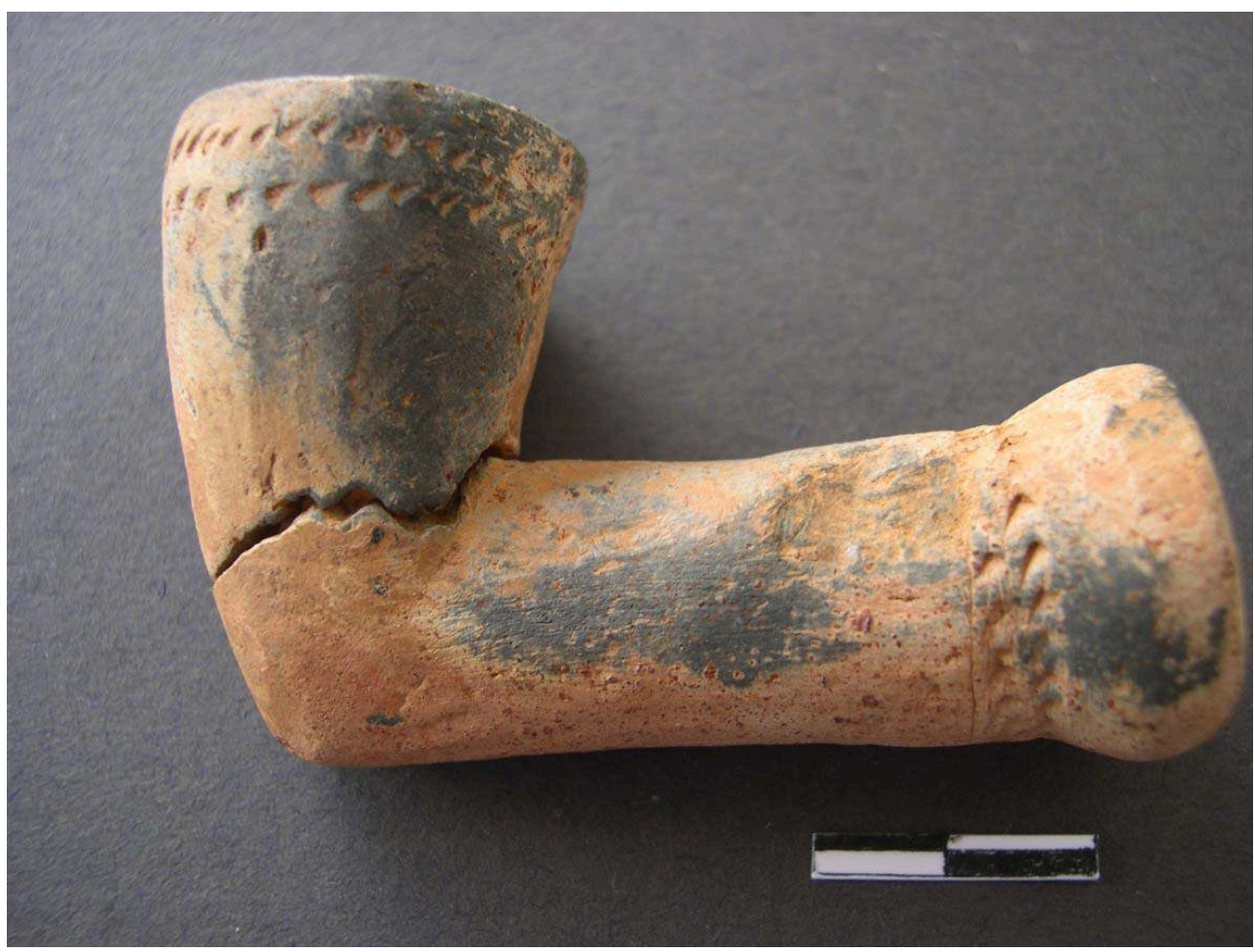

Pipe d'esclave retrouvée à Loyola. Elle témoigne, par sa morphologie et son décor, de la persistance, en Amérique, de modèles africains.

Phot. Y. Le Roux. (c) Y. Le Roux.

\section{Le quartier des esclaves}

L'importante population servile de Loyola constitue un véritable défi pour un établissement esclavagiste. Avec 500 personnes, le quartier des esclaves se présente plus comme un petit village que comme une simple rue "cases nègres". S'il faut en croire les sources écrites traitant de ce sujet, le marronnage, conséquence fréquente d'ateliers trop peuplés, n'a jamais concerné cet établissement. On peut conjecturer que cette situation est autant la conséquence du pouvoir de sujétion lié à la religion que de l'organisation matérielle de la vie des esclaves. Nourriture et logement, "faux frais » pour la plupart des habitants, étaient au contraire l'objet d'un soin particulier par les jésuites. On peut donc supposer qu'ils ont organisé cet espace de vie en respectant par exemple la cellule familiale comme en témoigne l'inventaire des esclaves retrouvé dans les archives des jésuites.

Le quartier des esclaves de Loyola est situé dans la partie basse du site, nommée La Savane. Ce vestige est aujourd'hui très altéré par des aménagements récents comme la construction de villas et le percement d'une importante voie urbaine. Malgré ces destructions, certaines zones, peu perturbées, restent aujourd'hui susceptibles d'être étudiées. Les cases d'esclaves, construites en matériaux périssables, ne seront pas 
faciles à retrouver. Seul le recours à une fouille en aire ouverte sur une très grande superficie présente quelques chances d'obtenir des résultats significatifs (fig. $\mathbf{n}^{\circ} 7$ ).

Figure 7

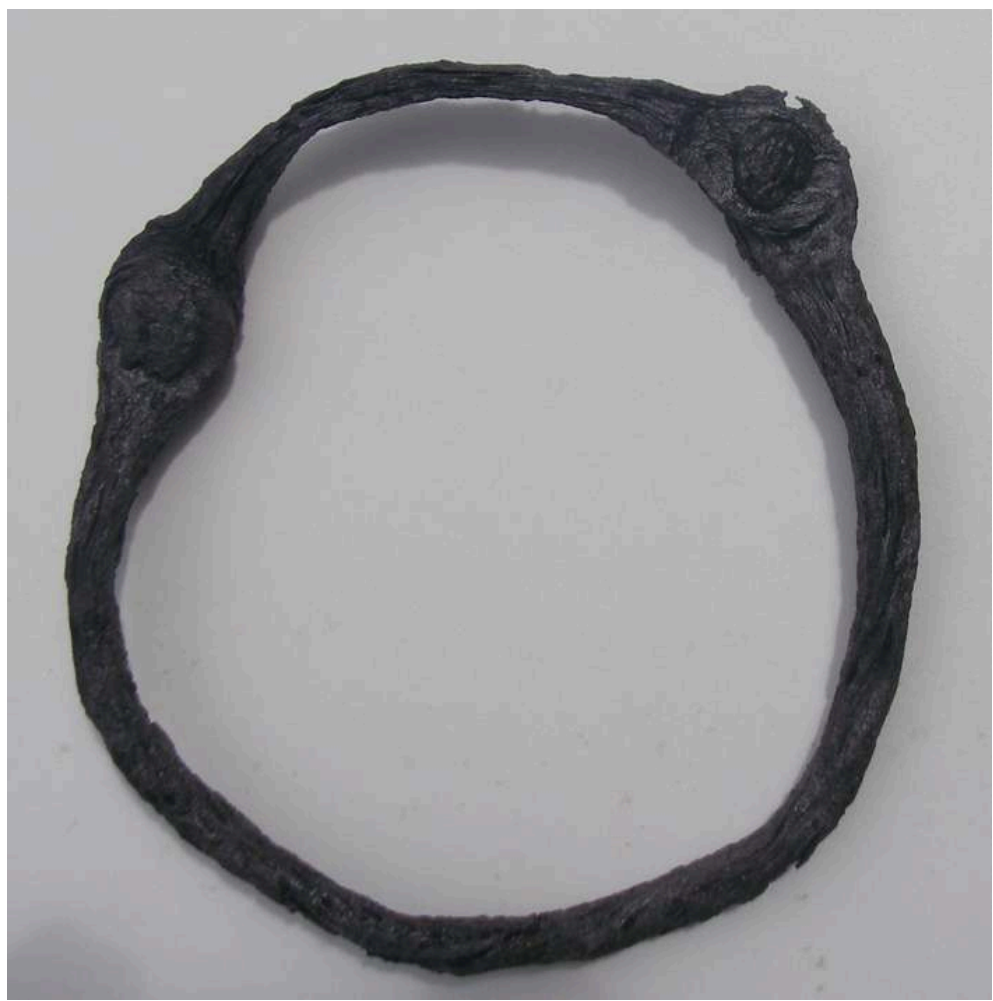

Collier de servitude. Cette entrave de cou en fer forgé provient du secteur de la maison de maître de Loyola.

Phot. L. Jodoin. (C) L. Jodoin.

\section{Le secteur religieux}

La chapelle de Loyola, Notre Dame de Grâce, est un bâtiment modeste, loin des fastes du baroque jésuite sud américain. Longue de 16 mètres large de 6, elle est à nef unique, sans transept. Le chœur est prolongé, à gauche, par une sacristie. Les carreaux de terre cuite qui recouvraient son sol ont été, en grande partie, démontés après abandon. On a cependant retrouvé de très grands fragments de carrelage en place dans la région du chœur. Ils permettent de situer l'emplacement de l'autel et la clôture du chœur. On sait que de nombreuses inhumations, tant de jésuites que d'habitants importants, ont été pratiquées dans cette église. Un seul sondage exploratoire a été, pour l'instant, effectué mais il s'est révélé stérile.

Attenant à la chapelle, s'étend le cimetière. La présence d'un cimetière aménagé au cœur d'une habitation est peu fréquente. On ne peut qu'y voir la volonté des jésuites de renforcer la dimension religieuse de leur établissement, attachant, au passage, leurs esclaves à cette "nouvelle terre de leurs ancêtres". Un sondage d'évaluation, de 4 mètres sur 3, a permis de confirmer l'usage de cette zone en révélant la présence de 10 fosses d'inhumations. Les conditions physico-chimiques des sols n'ont malheureusement pas permis la conservation des restes organiques. Les excavations 
des fosses ont cependant fourni des informations sur les orientations des corps et sur leur mode d'inhumation (dans un linceul et non dans un cercueil).

Cette année, un nouveau chantier archéologique sera ouvert pour reprendre l'étude de ce cimetière où reposent probablement plus de mille personnes (fig. $\left.\mathbf{n}^{\circ} \mathbf{8}\right)$.

Figure 8

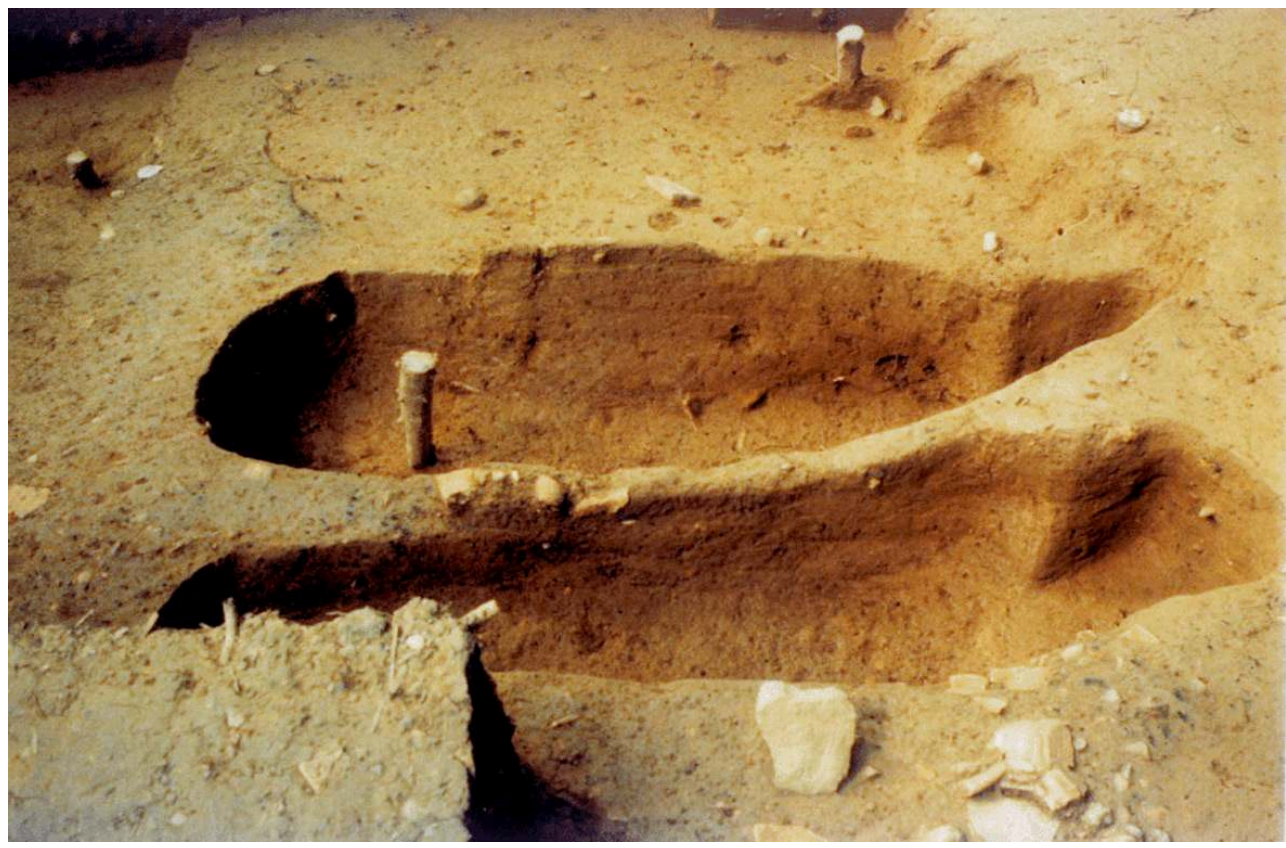

Fouilles du cimetière de Loyola. Fosses d'inhumations révélées à l'occasion d'un sondage. Les restes humains ont complètement disparu, détruits par l'action biologique et chimique du sol. On a pu constater que les défunts étaient portés en terre sans cercueil mais simplement enveloppés dans un linceul.

(C) E. Broine.

\section{Bâtiments agricoles et artisanaux}

Sur le territoire de Loyola, on a dénombré, à ce jour, une vingtaine de constructions plus ou moins importantes. Certaines sont reliées à la manufacture des denrées cultivées : sucre, café, coton... D'autres à des activités artisanales comme la forge, d'autres au stockage, au bétail... On imagine facilement que, sur ce vaste établissement, il existait aussi un nombre important de constructions en bois qui n'ont guère laissé de traces.

\section{Le Moulin à vent}

19 À moins d'un kilomètre de la maison de maître, un moulin à vent se dresse sur une colline d'une trentaine de mètres de hauteur. Cette belle tour, construite en pierre, porte une dédicace datée 1733. Le régime des vents, assez faibles et très irréguliers en Guyane, laisse supposer que ce moulin n'a pas beaucoup servi, il est d'ailleurs qualifié plus tard de "purement ostentatoire ». Le dégagement de cette construction, classée Monument historique, constitue la première étape d'une restauration, que l'on espère complète, de ce bâtiment sans équivalent en Guyane (fig. $\mathbf{n}^{\circ} \mathbf{9}$ ). 
Figure 9

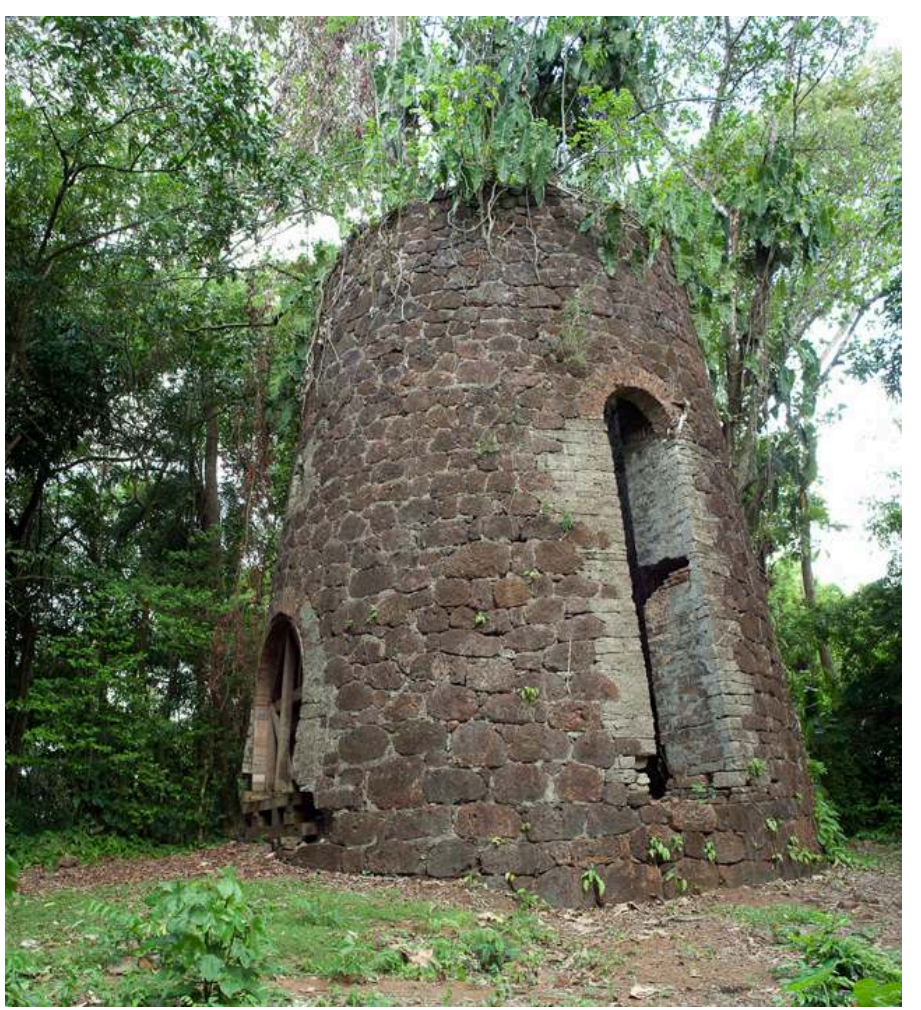

Vestige actuel du moulin à vent des jésuites de Loyola. Cette construction remonte à 1733.

(c) Y. Le Roux.

\section{Le moulin à bête?}

À une vingtaine de mètres, en contrebas de ce moulin, on a dégagé une plate-forme aménagée, interprétée comme l'emplacement d'un moulin à manège en bois. Il était mis en mouvement par des animaux, bœufs ou mulets. Ce moulin devait assurer l'essentiel du broyage des cannes destinées à la sucrerie.

\section{La chaufferie/sucrerie}

Un vaste ensemble de bâtiments, se déployant en façade sur près de 48 mètres avec une profondeur de 30 mètres, constitue les vestiges de la sucrerie des jésuites. Des documents nous apprennent que cette manufacture a été désaffectée en 1753. Effectivement, à l'emplacement de la chaufferie, nous n'avons pas retrouvé les équipages de chauffe qui devaient y être construits. Par contre, la partie opposée de ce bâtiment comporte trois fours, dont l'un a conservé la grille du foyer, que l'on interprète comme la vinaigrerie (ou distillerie) de l'habitation. Les textes de l'époque mentionnent que la production de sirop (mélasse) et de tafia (rhum de médiocre qualité) constituaient le principal débouché de cette manufacture dans sa période finale (fig. $\mathbf{n}^{\circ} \mathbf{1 0}$ ). 
Figure 10

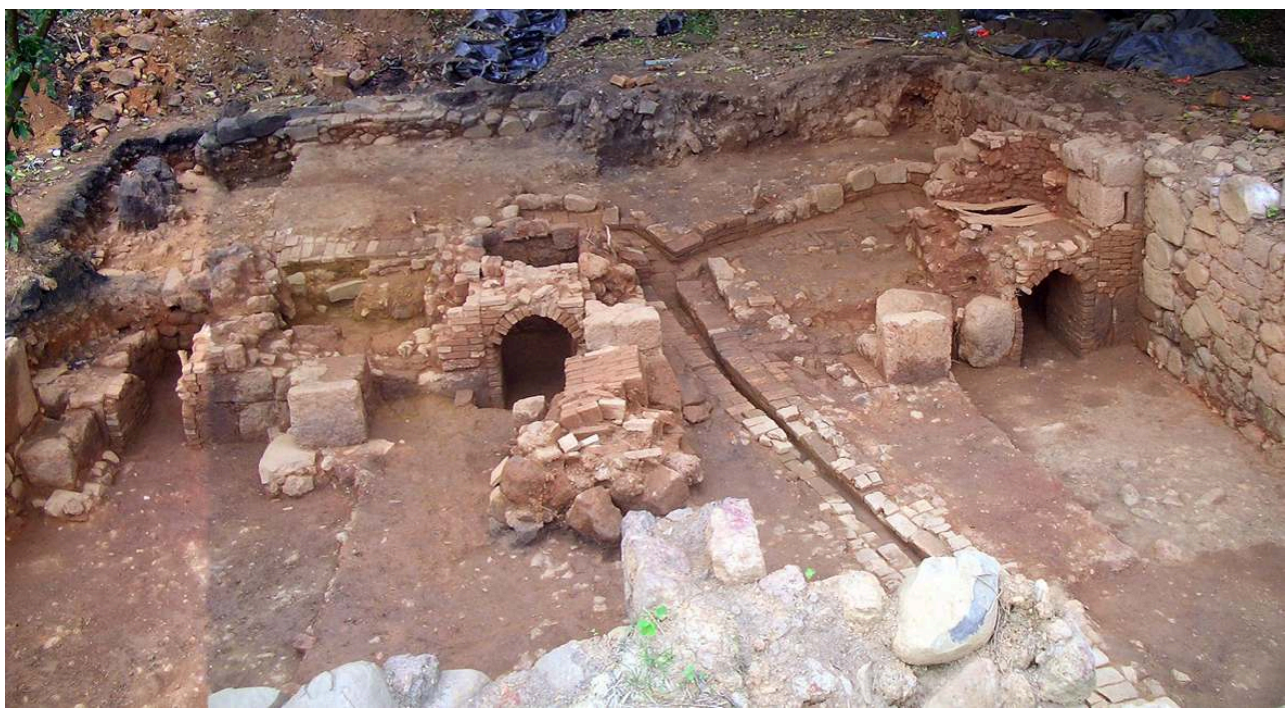

Vue de la fouille de la sucrerie, zone des fours de la vinaigrerie.

Phot. N. Cazelles. ㄷ N. Cazelles.

\section{La purgerie}

22 C'est un vaste édifice, de 32 mètres sur 12. Il n'a été, à ce jour, que partiellement étudié. Originellement, c'est dans ce bâtiment que s'effectuait la phase finale de la fabrication $\mathrm{du}$ sucre, le raffinage. Des textes anciens nous renseignent sur l'usage de la purgerie comme hangar à coton après l'abandon de la fabrication du sucre. En annexe de cette construction, on a retrouvé un petit bâtiment carré: l'étuve. C'est dans cette pièce confinée, alimentée par un poêle, que l'on mettait le sucre à sécher avant de l'expédier (fig. $\left.\mathbf{n}^{\circ} \mathbf{1 1}\right)$. 
Figure 11

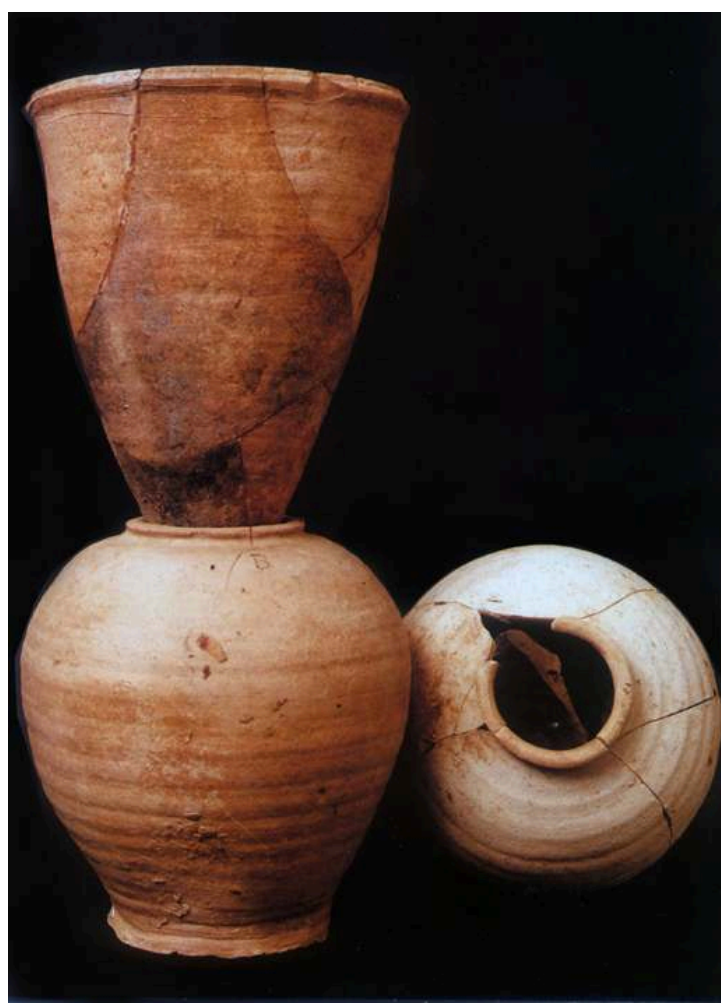

Poteries sucrières. Forme à sucre emboîtée dans un pot à mélasse ou de raffineur.

(c) Y. Le Roux.

\section{La caféterie/cacaoterie}

Deux bâtiments de 22 mètres de long, étagés sur les flancs de la colline du moulin à vent, ont été identifiés, en raison de leur forme allongée et de leur exposition plein sud, comme étant des ateliers de traitement et de séchage du café ou du cacao. Les jésuites ont été les premiers introducteurs de la culture du café en Guyane; en outre ils contrôlaient l'accès aux forêts de cacao, situées au sud de la Guyane. Vers 1736, ils produisaient la moitié du café et du cacao de la colonie.

\section{L'indigoterie}

Située à près de deux kilomètres du site principal, l'indigoterie des jésuites, fondée vers 1740 , utilisait l'eau du ruisseau de Rémire, près de son embouchure sur l'océan. Mais l'éloignement de cette manufacture s'explique aussi par sa production réputée nauséabonde. Les fouilles ont permis de retrouver une grande plate-forme de pierre regroupant toutes les installations de l'indigoterie, elle devait être protégée par une toiture végétale. Outre une aire de travail et un possible réservoir d'eau, on a excavé deux grands bassins identifiés comme une trempoire et une batterie. On note l'absence de la dernière cuve: le diablotin, l'indigo devait être recueilli dans la batterie par décantation (fig. $\mathbf{n}^{\circ} \mathbf{1 2}$ ). 
Figure 12

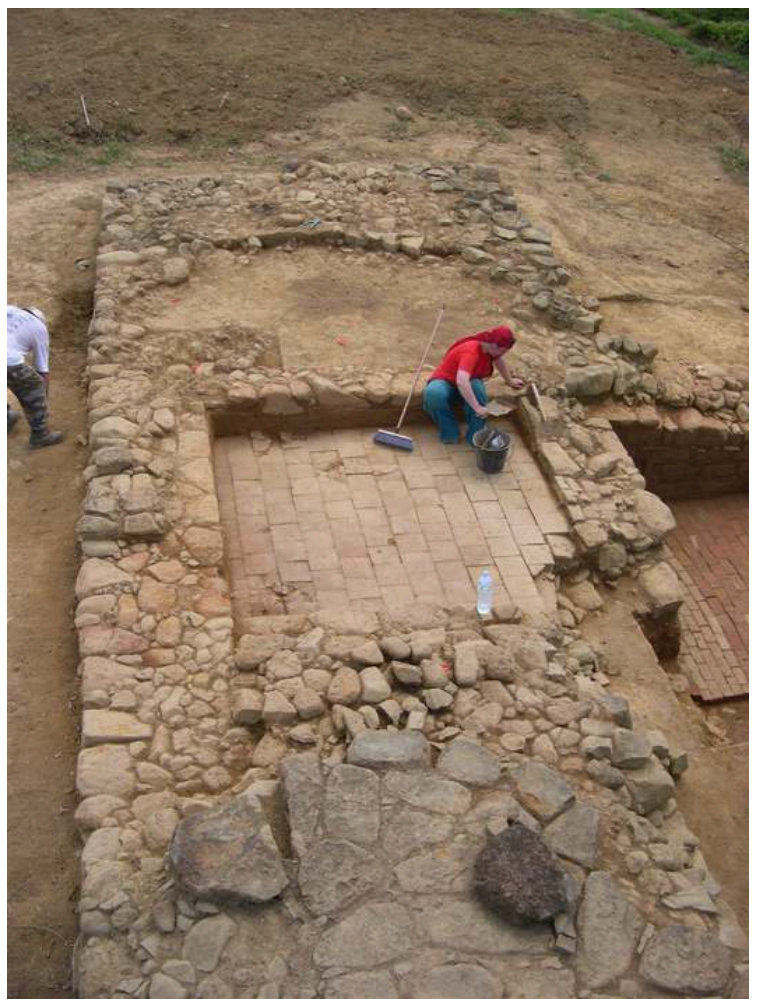

Fouille de l'indigoterie de Loyola. Mise à jour du bassin de la trempoire.

Phot. N. Cazelles. (c) N. Cazelles.

\section{La poterie}

Cet important atelier a été retrouvé en bordure des vastes marécages qui s'étendent derrière l'Hôtel de Ville de Rémire. Les jésuites ont exploité les gisements d'argile d'origine marine de cette région qui présentent d'excellentes qualités potières. Ils fabriquaient des briques et des carreaux de terre cuite mais aussi des poteries domestiques et industrielles. Les travaux archéologiques ont été limités à des sondages de reconnaissance de cette grande poterie qui possédait plusieurs fours. On a pu mettre également en évidence une production de poteries vernissées, ce qui est rare, voire inédit, dans les ateliers coloniaux de cette époque.

\section{La forge}

Rare exemple d'atelier colonial remontant à cette époque, la forge de Loyola est un bâtiment de 18 mètres sur 8 , à l'intérieur duquel on a retrouvé le soubassement du foyer. Un nombre important d'outils (plusieurs centaines), de lingots de fer, de mobilier en cuivre, a été exhumé dans le contexte de ce bâtiment (fig. $\mathbf{n}^{\circ} \mathbf{1 3}$ ). 
Figure 13

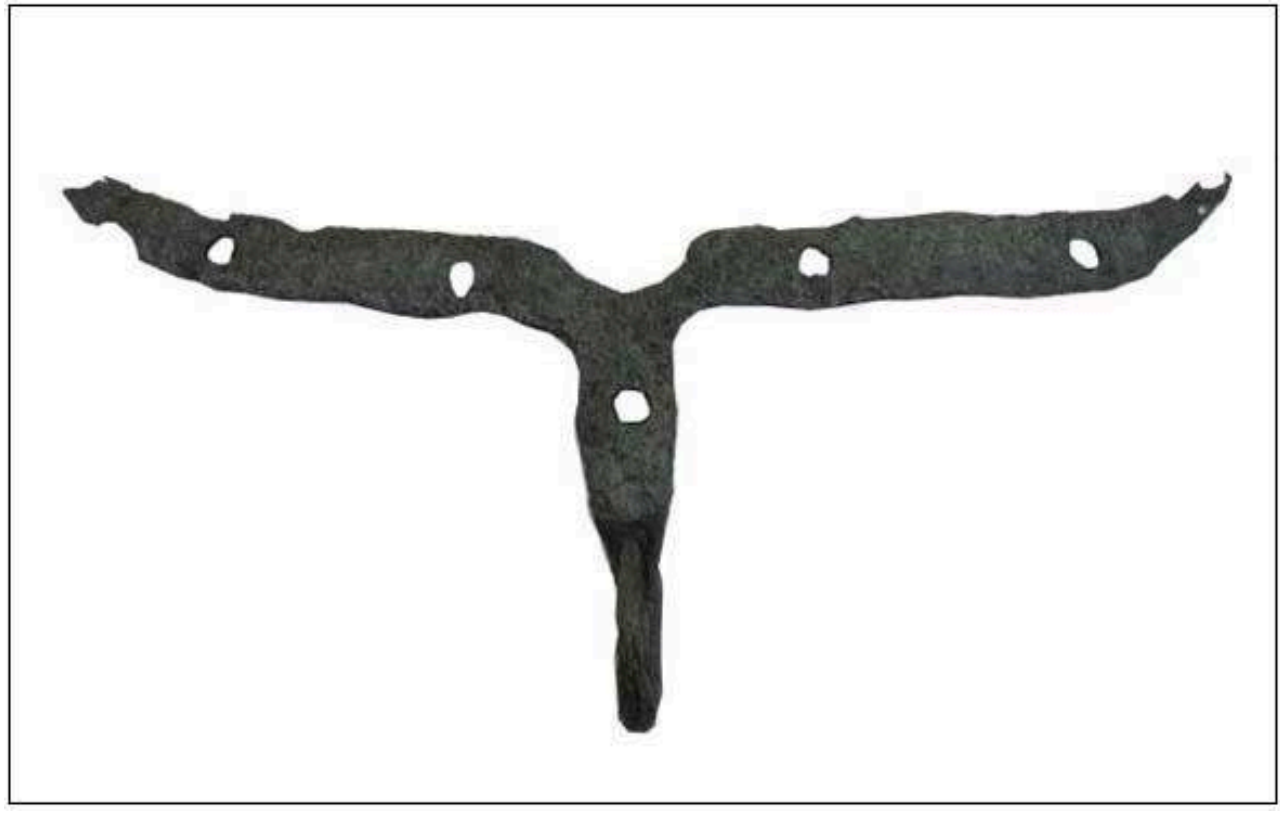

Crochet de fer, avec son système de fixation, retrouvé dans la forge de Loyola.

Phot. Y. Le Roux. @ Y. Le Roux.

27 Le magasin, construction de 17 mètres sur 8 mètres, servait à stocker les denrées nécessaires à l'habitation. Cette construction est en pierre, sur deux niveaux. L'étage, probablement en charpente, était accessible par une rampe. Non encore fouillé, ce bâtiment devrait faire l'objet d'une opération archéologique à partir de 2011.

\section{Les circulations, l'approvisionnement en eau}

À Loyola, on a mis au jour certains passages aménagés, des caniveaux, des voies de circulation. La plupart de ces aménagements sont des dallages de roches. Même si le pillage des matériaux a sérieusement perturbé la plupart de ces vestiges, ils conservent encore une lisibilité suffisante pour en reconstituer, au moins, le tracé initial.

Dépourvue de puits, de criques accessibles et permanentes, l'habitation Loyola était approvisionnée par un aqueduc qui captait l'eau d'une source située à 300 mètres de l'établissement. Les travaux archéologiques ont permis de comprendre la configuration de cette construction, depuis la zone de captage, jusqu'à l'arrivée des eaux sur l'habitation.

30 L'arrivée et la distribution de l'eau, à hauteur de la cuisine et de la maison de maître, sont malheureusement très ruinées ou détruites, ce qui explique qu'elles n'aient pas pu être complètement élucidées aujourd'hui.

\section{Bilan des travaux scientifiques et des opérations de mise en valeur réalisés à Loyola}

La situation a beaucoup évolué depuis la découverte des ruines de Loyola, en 1988, par Patrick Huard, alors que cette grande habitation avait totalement disparu du paysage 
guyanais. L'année suivante, le plan d'occupation des sols de Rémire-Montjoly délimitait une première zone de protection, malheureusement insuffisante, puisqu'en 1991, des travaux d'aménagement menaçaient la partie basse du site. Il était alors urgent d'intervenir pour sauver Loyola, menacé de disparition pure et simple par la réalisation d'importants programmes immobiliers. Une procédure d'inscription de l'ensemble des vestiges, au titre de l'inventaire supplémentaire des monuments historiques, en 1993', allait marquer un premier arrêt des tentatives des promoteurs immobiliers. Un bornage, délimitant les zones d'intérêt archéologique, fut alors effectué. Cette mesure permit de suspendre certains projets de construction, mais seule l'acquisition du site, en 2008, par le Conservatoire du Littoral a permis la mise à l'abri définitive de cet ensemble. Dès lors, une voie pleine de promesse pour l'ouverture du site au public était ouverte.

Le premier chantier archéologique a démarré en 1994, il avait pour objet d'évaluer ces vestiges et de produire des études scientifiques portant sur les structures et le mobilier recueilli au cours des fouilles. Ces recherches ont été menées dans le cadre d'une association archéologique guyanaise (l'APPAAG) confortées par la collaboration du centre d'archéologie médiévale de l'université de Caen (Professeur Claude Lorren) jusqu'en 1998. Depuis 1996 et jusqu'à aujourd'hui, sans interruption, les recherches archéologiques de Loyola ont bénéficié de la collaboration de l'Université Laval à Québec: apports d'étudiants, analyses de laboratoire, publications sous la responsabilité du professeur Réginald Auger (fig. $\mathbf{n}^{\circ} \mathbf{1 4}$ ).

Figure 14

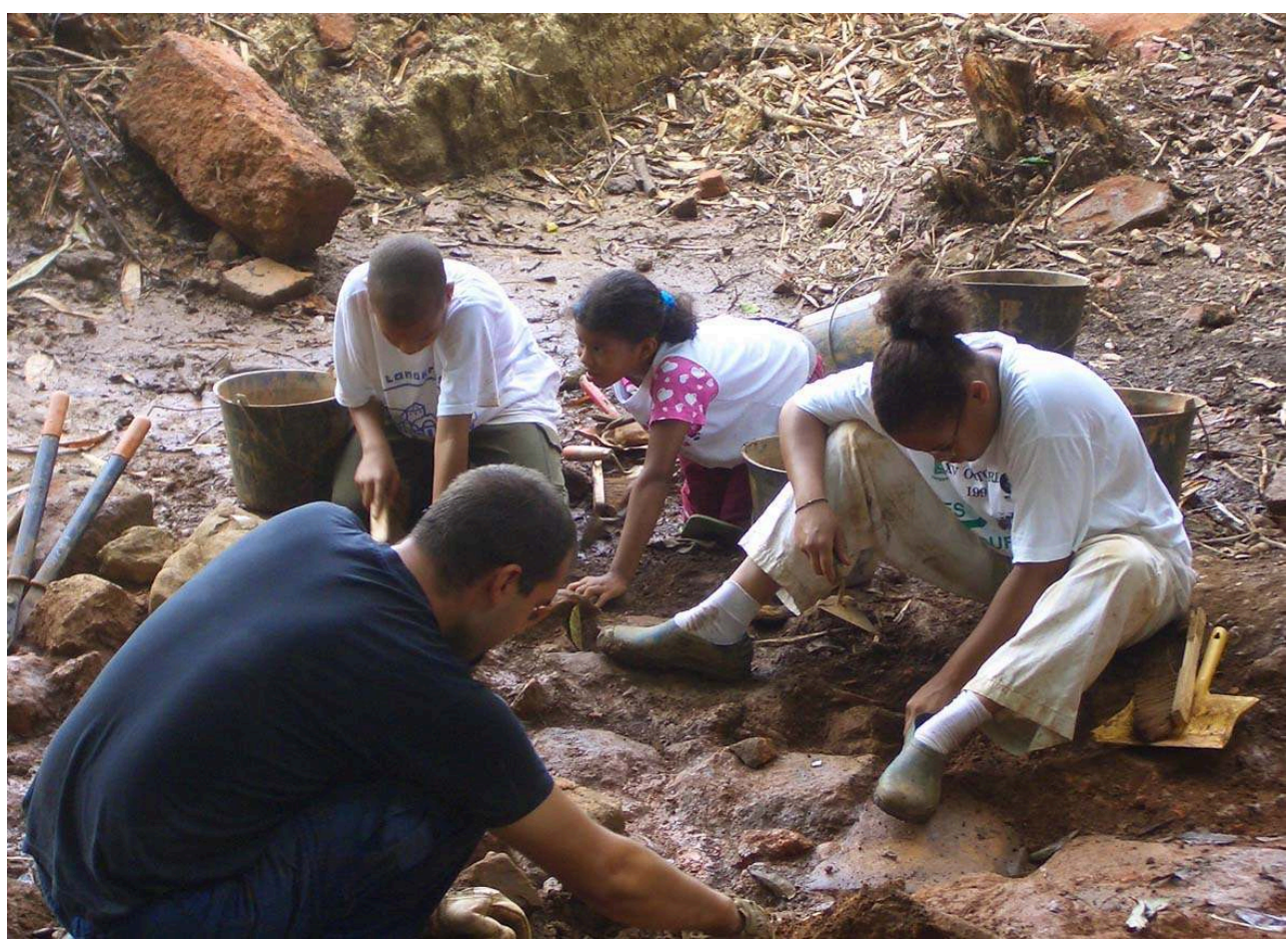

Jeunes fouilleurs au travail dans le secteur de la sucrerie en 2005.

Phot. N. Cazelles. ( ) N. Cazelles

33 En 2000, les recherches sur ce site étaient interrompues à cause du refus des propriétaires de poursuivre des fouilles sur ces terrains. Cette pause forcée fut mise à 
profit pour étudier le fruit de ces six années de fouilles programmées et pour se déployer sur d'autres sites plus ou moins éloignés (de 300 mètres à 2 kilomètres), mais tous dépendants de cet immense domaine de plus de 1000 hectares. Pendant cette première décennie, les travaux ont ainsi porté sur la source et son aqueduc, la poterie, la caféterie, le moulin à vent, l'indigoterie, le site de Quincy, enfin la grande sucrerie de Loyola : ses moulin à vent et moulin à bête, sa vinaigrerie et sa chaufferie.

À ce jour, plus de cinquante rapport, études, articles, ouvrages constituent la bibliographie du site de Loyola. Neuf mémoires de maîtrise (Paris 1 et Université Laval) ont été soutenus, portant sur différents aspects de cet ensemble; trois doctorats, s'appuyant en grande partie sur les résultats des fouilles effectuées sont en cours d'achèvement (2011 et 2012). Tous ces travaux ont été synthétisés dans un livre à grande diffusion : Loyola, les jésuites et l'esclavage, véritable monographie de cette habitation. L'accueil rencontré par cette publication montre l'intérêt du public pour cet important vestige qui vit désormais une véritable renaissance grâce aux travaux dont il est l'objet.

En 2009, le Conservatoire du Littoral, nouveau propriétaire des lieux, décidait un programme de restauration et de mise en valeur des vestiges de la maison de maître. Une archéologue de l'Université Laval, Catherine Losier, était dépêchée sur place pour effectuer des fouilles préventives qui ont révélé des aménagements inédits dans le secteur de la chapelle. Au cours de l'été 2010, Réginald Auger, Yannick Le Roux et trois étudiants de Québec ont effectué une opération archéologique destinée à étudier les écoulements d'eaux sur le site à l'époque des jésuites : aqueduc, caniveaux.

Ces travaux ont permis d'orienter les opérations de restauration et de mesurer sur le terrain la nécessité de reprendre, en profondeur, les recherches archéologiques sur cet ensemble aussi complexe que riche en vestiges. Aujourd'hui bien des questions subsistent sur la chronologie, les fonctions des bâtiments, le mobilier, la vie des esclaves... Les sources écrites, relativement rares, et en tout cas très lacunaires, ont montré leurs limites. Seules de nouvelles découvertes archéologiques permettront de faire évoluer nos connaissances sur cette page d'histoire. Un magasin proche de la maison de maître, la chapelle dans son entier, le cimetière, la purgerie, etc, vont faire l'objet d'études archéologiques et de mise en valeur exhaustives. Le site de Quincy, repéré comme l'ancien établissement des jésuites, remontant à la fin du XVII ${ }^{\mathrm{e}}$ siècle, doit être également étudié. Le quartier des esclaves, malgré des perturbations dommageables, doit être étudié dans le cadre de l'aménagement d'un parking à l'entrée du site (fig. $\left.\mathbf{n}^{\circ} \mathbf{1 5}\right)$. 
Figure 15

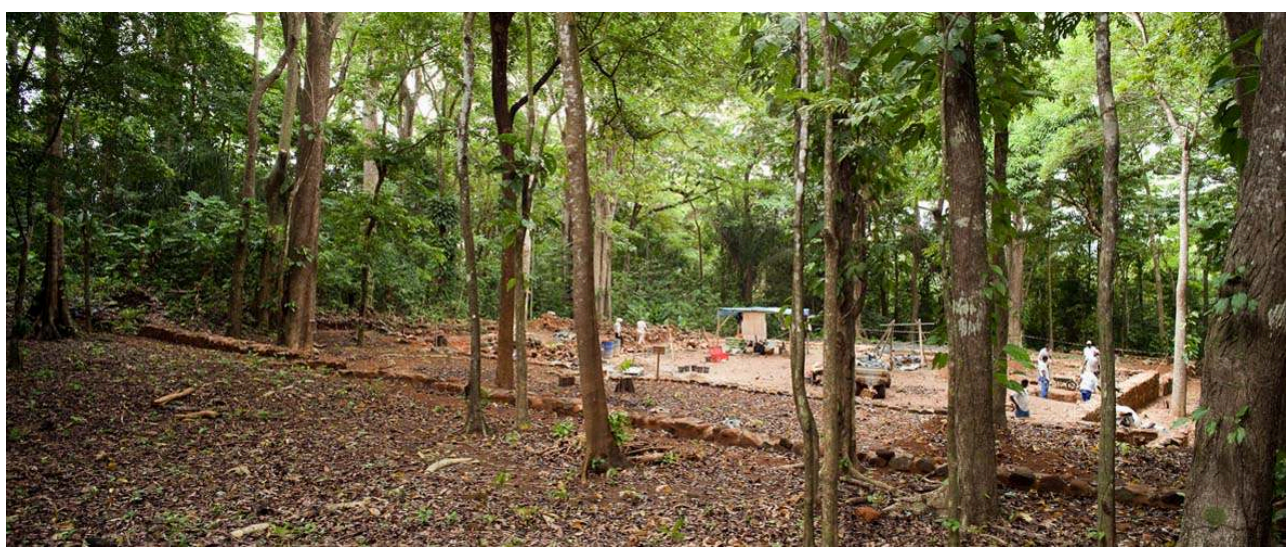

Vue générale du chantier de restauration en 2010.

Phot. A. Le Roux. (c) A. Le Roux.

37 Les travaux de restauration, entrepris par le Conservatoire du Littoral, avec l'aval de l'administration des Monuments historiques (DRAC Guyane), ont d'abord porté sur la consolidation des terrasses par le remontage des murs de soutien. La circulation des eaux, très importante sur ce site en pente, a été en grande partie maîtrisée par la pose de drains enterrés. Les restaurations des murs de la cuisine et de l'hôpital au niveau des arases permettent de recouvrir la visibilité de ces deux bâtiments importants. La restauration des escaliers d'accès aux terrasses, la remise en état du jardin de la cour carrée, avec la restauration des allées, composent déjà un ensemble paysager en clairière bordée par des grands arbres (bois diable). La chapelle et la maison de maître doivent être, à leur tour, mises en valeur. L'emplacement du cimetière sera entièrement dégagé. L'aménagement d'un chemin de randonnée, traversant le site jusqu'à la mer, est en cours de réalisation.

La restauration du moulin à vent, avec sa toiture et ses ailes, la consolidation des ruines de la sucrerie et de l'indigoterie sont programmées dans les années à venir.

Le Conservatoire national d'histoire naturelle a présenté un projet destiné à utiliser une partie du site pour présenter les plantes en usage dans les sociétés traditionnelles de la Guyane mais aussi de présenter celles qui étaient utilisées à l'époque d'activité de l'habitation.

À terme, on espère que la Guyane, dépourvue à ce jour de sites esclavagistes aménagés et ouverts en permanence au public, pourra disposer, grâce à Loyola, d'un lieu répondant à cette attente d'histoire et de mémoire qui se manifeste aujourd'hui dans la population.

\section{Bibliographie}

AUGER, Réginald, LE ROUX, Yannick and BAIN, Allison. Loyola. A 17th-18th Century jesuit Sugar-Processing Habitation in French Guyana. 40 th Annual Conference on Historical and Underwater Archaeology. Williamsburg, Virginia, 2007.

BARONE, Eglée, MICHEL, P. «Les habitations jésuites de Saint-Régis et Le Maripa en Guyane ». Dans Le sucre, de l'Antiquité à son destin Antillais, sous la direction de Danièle Bégot. Paris : éd du CTHS, 2000, p. 103-120. 
BIGOT, Viviane. L'habitation Loyola depuis le départ des jésuites: une lecture de l'occupation des sols à partir des archives historiques (1764-1938). Mémoire de maîtrise, Université de Paris I Sorbonne, 2004, p. 165.

BIGOT, Viviane. "L'habitation Loyola dite du Moulin à Vent d'après les sources archivistiques : la transmission du passé colonial en Guyane Française ». L'histoire de la Guyane. Matoury : Ibis Rouge éditions, 2006, p. 599-617.

CAZELLES, Nathalie. Le site du Moulin à vent. Rapport de synthèse de fouille programmée. 2004-2005-2006. SRA-Guyane, $110 \mathrm{p}$.

CHOUINARD, Alain. "Archéologie et archéométallurgie de la forge et des forgerons de l'habitation Loyola en Guyane ». Cahiers d'archéologie du Celat 2. Québec : CELAT, Université Laval, 2001. ํำ11, 2001, 112 p.

COLLOMB, Gérard. "Les Indiens de la Sinnamary». Journal du père Jean de la Mousse. (1684-1691). Paris : éd Camdeigne, 2006, 318 p.

CROTEAU, Nathalie. Loyola, une habitation guyanaise sous l'Ancien Régime. Programme de mise en valeur des vestiges archéologiques. Mémoire de maitrise; Faculté des Lettres, Université Laval, Québec, 1999, 137 p.

CROTEAU, Nathalie. «L'Habitation Loyola: un rare exemple de prospérité en Guyane française ». Journal of Caribbean Archeology. Special publication, $\mathrm{n}^{\circ}$ 1, p. 68-80.

GOUPY DES MARETS, Jean. Voyage de Goupy aux Iles d'Amérique et aux côtes d'Afrique. 1675-1676. 1687-1690 (deuxième voyage). Rouen: bibliothèque municipale, collection Montbret, manuscrit 2436.

Copie ancienne : Bibliothèque Franconie, Cayenne. Ms. 200.

HURAULT, Jean. Français et Indiens de Guyane. 1604-1972. Cayenne: Guyane Presse diffusion, 1989, $222 \mathrm{p}$.

LAPRAY, Isabelle. Association d'entretien et de gestion de sites patrimoniaux dans l'île de Cayenne. Université Lumière, Lyon 2. DESS développement et promotion des aménagements touristiques. Lyon, 1998, 171 p.

LE ROUX, Yannick. Rémire aux XVII et ${ }^{e}$ XVIII ${ }^{e}$ siècles. Équinoxe, n¹8, 1984, p. 69-78.

LE ROUX, Yannick. L'habitation Poulain à Rémire. Mémoire de l'EHESS, Paris, 1986, 370 p.

LE ROUX, Yannick, ROSTAIN, Stephen. L'archéologie. Cayenne : éd. Saga, 1989, 29 p.

LE ROUX, Yannick. L'Habitation guyanaise sous l'Ancien Régime. Étude de la culture matérielle. Thèse de doctorat, École des Hautes Études. Paris, 1994, 863 p.

LE ROUX, Yannick. "L'archéologie de la période coloniale ». L'Archéologie en Guyane. APPAAG. Cayenne, 1997, p. 161-177.

60 LE ROUX, Yannick, LORREN, Claude, BROINE, Claude. Loyola. 1996. Rapport archéologique. Cayenne, SRA, 1996, 22 p.

61 LE ROUX, Yannick. Loyola. Bilan scientifique régional. Guyane, Cayenne, 1996, p. 34-39.

2 LE ROUX, Yannick. L'habitation Loyola à Rémire, Guyane française. Rapport de synthèse sur l'opération de fouille programmée triennale. SRA Cayenne, 1998, 180 p.

3 LE ROUX, Yannick. "L'Habitation Loyola à Rémire, l'archéologie coloniale, pour l'histoire ». L'histoire de la Guyane. Matoury : Ibis Rouge éditions, 2006, p. 599-617.

LE ROUX, Yannick, AUGER, Réginald, CAZELLES, Nathalie. Loyola, les jésuites et l'esclavage... Québec: Presses de l'Université du Québec, 2009, 294 p. 


\section{NOTES}

1. - Vestiges de l'habitation des jésuites Loyola, site de Rémire : Inscription en totalité, y compris leur terrain d'assiette. ISMH par arrêté $\mathrm{n}^{\circ} 1860 \mathrm{du}$ 11/10/1993. Moulin à vent, CD 2, route de Rémire : classé en totalité, CLMH par arrêté n94-146 du 18/10/1994.

\section{RÉSUMÉS}

Cette communication est une présentation générale des résultats des interventions archéologiques conduites à Loyola, une habitation exploitée par les jésuites en Guyane, à partir de 1668 et jusqu'à leur interdiction en 1763. L'habitation Loyola, située à Rémire, à $10 \mathrm{~km}$ de Cayenne, couvre une superficie d'un peu plus de 1000 hectares. Jusqu'à 500 esclaves y travaillèrent à produire des biens dont les revenus finançaient le grand projet des religieux en Amérique : l'évangélisation des Amérindiens par le biais des missions. Cet établissement illustre, de façon éloquente, la relation particulière qu'entretint l'église catholique avec le système esclavagiste. Nous présentons un bref historique de cette habitation et de la place qu'occupaient les missionnaires dans cette aventure coloniale. Les recherches ont mis au jour la maison de maître et ses dépendances, la chapelle et le cimetière, une forge, une poterie, une manufacture sucrière complète (moulins, chaufferie, purgerie et étuve) une caféterie, une indigoterie... Un très abondant mobilier céramique, verrier et métallique a été recueilli au cours des opérations archéologiques; il permet de préciser les conditions de vie et les relations commerciales de cette habitation, exceptionnelle par ses dimensions mais représentative de l'économie coloniale à cette 
époque. Une entreprise de mise en valeur du site est actuellement engagée, elle vise à révéler au public un lieu illustrant, de façon éloquente, la mémoire de l'esclavage en Guyane.

This communication is an overview of the results of our archaeological work at Loyola, a sugar plantation owned by the Jesuits in French Guiana from 1668 until its ban in 1763. The Loyola plantation in Rémire is located at $10 \mathrm{~km}$ from the fortified city of Cayenne and it covers an area just over 1000 hectares. At one point up to 500 slaves worked at the plantation to produce goods which, when sold to France and other French colonies, brought benefits that were used to support the Jesuit's missions which aimed to spread evangelization to South American Natives. Loyola illustrates with eloquence the relationship between the Catholic Church and slavery. We present a brief history of this house and the place missionaries occupied in the colonial adventure. We discuss the role they played in the economy of this French colony, considered, rather marginal, in comparison to what is revealed at other the Caribbean examples. Our research has uncovered the master's house and its outbuildings, a chapel and its cemetery, a blacksmith shop, a pottery, as well as remains of the sugar production such as the mills, boiler and dryer. We also have evidence of coffee production, indigo etc. We have also recovered a large collection of locally made ceramics used for sugar production; imported domestic ceramic and glass and metal farming tools. A project of development of the site is currently engaged, it aims at revealing with the public a illustrating place, in an eloquent way, the memory of slavery in Guyana.

\section{INDEX}

Mots-clés : Ancien Régime, archéologie, caféterie, cimetière, esclavage, habitation, indigoterie, jésuites, Guyane, religion, sucrerie

\section{AUTEUR}

YANNICK LE ROUX

Professeur agrégé, docteur en histoire et civilisation (EHESS), Président de l'APPAAG rorota@wanadoo.fr 